\title{
Lagrangian Duality for Multiobjective Programming Problems in Lexicographic Order
}

\author{
X. F. Hu ${ }^{1}$ and L. N. Wang ${ }^{2}$ \\ ${ }^{1}$ School of Information Engineering, Chongqing City Management Vocational College, Chongqing 401331, China \\ ${ }^{2}$ Chongqing Water Resources and Electric Engineering College, Chongqing 402160, China \\ Correspondence should be addressed to L. N. Wang; wanglncq@yeah.net
}

Received 27 April 2013; Revised 19 September 2013; Accepted 22 September 2013

Academic Editor: Shawn X. Wang

Copyright (c) 2013 X. F. Hu and L. N. Wang. This is an open access article distributed under the Creative Commons Attribution License, which permits unrestricted use, distribution, and reproduction in any medium, provided the original work is properly cited.

This paper deals with a constraint multiobjective programming problem and its dual problem in the lexicographic order. We establish some duality theorems and present several existence results of a Lagrange multiplier and a lexicographic saddle point theorem. Meanwhile, we study the relations between the lexicographic saddle point and the lexicographic solution to a multiobjective programming problem.

\section{Introduction}

Duality assertions are very important in optimization researches from the theoretical as well as from the numerical point of view. Duality theorems in mathematical programming establish typical connections between a constrained minimization problem and a constrained maximization problem. The relationship is such that the existence of a solution to one of these problems ensures the existence of a solution to other, both having the same optimal value. In the past centuries, many authors have studied the duality problems of vector optimization problems; see, for example, [1-11] and reference therein.

On the other hand, it is well known that partial order plays an important role in multiobjective optimization theory. But partial efficient points are usually large, so that one needs certain additional rules to reduce them. One of the possible approaches is to utilize the lexicographic order, which is introduced by the lexicographic cone. The main reason is that the lexicographic order is a total ordering and it can overcome the shortcoming that not all points can be compared in partial order. The lexicographic order has been investigated in connection with its applications in optimization and decision making theory; see, for example, [12-19] and references therein. However, the lexicographic cone is neither open nor closed. Note that a lot of results for vector optimization problems are gotten under the assumption that the ordering cone is open or closed. Therefore, it is valuable to investigate multiobjective optimization problems in the lexicographic order. Konnov [12] first discussed the vector equilibrium problems in lexicographic order. Recently, Li et al. [13] studied the minimax inequality problem and have obtained minmax theorems in the lexicographic order.

The rest of the paper is organized as follows. In Section 2, we first recall some definitions and their properties. In Section 3, with respect to the lexicographic order, we first establish weak duality theorem and the Lagrangian multiplier rules for a multiobjective programming. In Section 4, we investigate a lexicographic saddle point of a vector-valued Lagrangian function and discuss the connection between the lexicographic saddle point and the lexicographic solution to a multiobjective programming problem.

\section{Preliminaries and Notations}

Throughout this paper, unless otherwise specified, let $X$ be an arbitrarily nonempty subset of a topology space $Y$ and $\mathbb{R}^{\ell} n$ dimensional Euclidean space. Let $\mathbb{R}_{+}^{\ell}:=\left\{x \in \mathbb{R}^{\ell}: x_{i} \geq 0, \forall i\right\}$. Let $\mathscr{L}\left(\mathbb{R}^{m}, \mathbb{R}^{n}\right)$ be the space of continuous linear operator from $\mathbb{R}^{m}$ to $\mathbb{R}^{n}$ and $\mathscr{L}^{+}\left(\mathbb{R}^{m}, \mathbb{R}^{n}\right):=\left\{\Lambda \in \mathscr{L}\left(\mathbb{R}^{m}, \mathbb{R}^{n}\right)\right.$ : $\left.\Lambda\left(\mathbb{R}_{+}^{m}\right) \subset \mathbb{R}_{+}^{n}\right\}$. By $0_{\ell}$ denote the zero vector of $\mathbb{R}^{\ell}$. 
For convenience, we set $I_{n}=\{1,2, \ldots, n\}$. As usual, for any $v \in \mathbb{R}^{n}$ and $i \in I_{n}$, $v_{i}$ will denote the $i$ th coordinate of $v$ with respect to the canonical basis $\left\{e_{1}, e_{2}, \ldots, e_{n}\right\}$. The lexicographic cone of $\mathbb{R}^{n}$ is defined as the set of all vectors whose first nonzero coordinate (if any) is positive:

$$
\begin{array}{r}
C_{\text {lex }}=\{0\} \cup\left\{v \in R^{n}: \exists i \in I_{n} \text { such that } v_{i}>0 ;\right. \\
\left.\nexists j \in I_{n}, j<i \text { such that } v_{j} \neq 0\right\} .
\end{array}
$$

Note that the lexicographic cone $C_{\text {lex }}$ is neither closed nor open. However, it is convex, pointed and $C_{\text {lex }} \cup\left(-C_{\text {lex }}\right)=\mathbb{R}^{n}$. Thus the binary relation defined for any $u, v \in \mathbb{R}^{n}$ by

$$
u \leq_{\text {lex }} v \Longleftrightarrow u \in v-C_{\text {lex }}
$$

is a total order on $\mathbb{R}^{n}$ (i.e., it is reflexive, transitive, antisymmetric and any two vectors are comparable). The binary relation induced by $C_{\text {lex }}$ is called a lexicographic order.

Now we recall the definitions of efficient points of a nonempty subset in the lexicographic order.

Definition 1 (see $[13,14]$ ). Let $Z \subset \mathbb{R}^{n}$ be a nonempty subset.

(i) A point $z_{0} \in Z$ is called a lexicographic maximal point of $Z$ if $Z \subset z_{0}-C_{\text {lex }}$; that is, for any $z \in Z, z \leq_{\operatorname{lex}} z_{0}$; by $\max _{\text {lex }} Z$, we denote the set of all lexicographic maximal points of $Z$.

(ii) A point $z_{0} \in Z$ is called a lexicographic minimal point of $Z$ if $Z \subset z_{0}+C_{\text {lex }}$; that is, for any $z \in Z, z_{0} \leq_{\text {lex }} z$; by $\min _{\text {lex }} Z$, we denote the set of all lexicographic minimal points of $Z$.

Obviously, if $\max _{\mathrm{lex}} Z \neq \emptyset, \max _{\mathrm{lex}} Z$ is a single point set and so is $\min _{\text {lex }} Z$.

Lemma 2 (see [13]). If $Z \subset \mathbb{R}^{n}$ is a nonempty compact set, then $\min _{\text {lex }} Z \neq \emptyset$ and $\max _{\text {lex }} Z \neq \emptyset$.

Proposition 3. If $C, D \subset \mathbb{R}^{n}$ are two nonempty compact subsets, then

(i) $\min _{\text {lex }}(C+D)=\min _{\text {lex }}(C)+\min _{\text {lex }}(D)$,

(ii) $\max _{l e x}(C+D)=\max _{l e x}(C)+\max _{l e x}(D)$.

Proof. It suffices to verify (i) since (ii) is evident by $\max _{\text {lex }}(C)=-\min _{\text {lex }}(-C)$. Indeed, let $\bar{x}=\min _{\text {lex }}(C)$ and $\bar{y}=\min _{\text {lex }}(D)$. Then $\bar{x} \in C$ and $\bar{x} \leq_{\text {lex }} x$, for all $x \in C$; $\bar{y} \in D$ and $\bar{y} \leq_{\operatorname{lex}} y$, for all $y \in D$. Hence, $\bar{x}+\bar{y} \in C+D$ and $\bar{x}+\bar{y} \leq_{\operatorname{lex}} x+y$ for all $x \in C$, and $y \in D$. Namely, $\{\bar{x}+\bar{y}\}=\min _{\text {lex }}(C+D)$ and the proof is complete.

Definition 4. A vector-valued mapping $g: X \rightarrow \mathbb{R}^{\ell}$ is called lower semicontinuous if, for any $z \in \mathbb{R}^{\ell}$, the set $\{x \in X$ : $\left.g(x) \in z-\mathbb{R}_{+}^{\ell}\right\}$ is closed in $X$.

Definition 5. A vector-valued mapping $g: X \rightarrow \mathbb{R}^{\ell}$ is called convex on $Y$ if and only if, for any $x_{1}, x_{2} \in X, t \in[0,1]$, and $i=1,2, \ldots, \ell, g_{i}\left(t x_{1}+(1-t) x_{2}\right) \leq t g_{i}\left(x_{1}\right)+(1-t) g_{i}\left(x_{2}\right)$.
Consider the following multiobjective programming problem:

$$
\begin{array}{ll}
\min _{\text {lex }} & f(x)=\left(f_{1}(x), f_{2}(x), \ldots, f_{n}(x)\right)^{T} \\
\text { s.t. } & g(x) \in-R_{+}^{m}, \\
& x \in X,
\end{array}
$$

where $f_{i}: X \rightarrow \mathbb{R}(i=1,2, \ldots, n)$ and $g(x)=$ $\left(g_{1}(x), g_{2}(x), \ldots, g_{m}(x)\right)^{T}$, and $g_{j}: X \rightarrow \mathbb{R}(j=1,2, \ldots, m)$.

Let $E$ denote the set of all feasible points for the multiobjective optimization problem (MP); that is,

$$
E=\left\{x \in X: g(x) \in-R_{+}^{m}\right\} .
$$

And let $E_{i}$ denote the set of all minimal points for $f_{i}$ on $E$; that is,

$$
E_{i}=\left\{x \in E: f_{i}(x)=\min _{y \in E} f_{i}(y)\right\}, \quad \text { for } i=1,2, \ldots, n .
$$

Then, we write $f(E)=\bigcup_{x \in E} f(x)$. Throughout this paper, we always assume that $E \neq \emptyset$ and $E_{i} \neq \emptyset(i=1,2, \ldots, n)$.

A vector-valued Lagrangian function for (MP) is defined from $E \times R^{n \times m} \rightarrow R^{n}$ by

$$
L(x, \Lambda)=f(x)+\Lambda g(x),
$$

where $\Lambda \in \mathscr{L}^{+}\left(R^{m}, R^{n}\right)$; that is, $\Lambda_{n \times m}=\left(\lambda_{1}, \lambda_{2}, \ldots, \lambda_{n}\right)^{T}$; $\lambda_{i}=\left(\lambda_{i 1}, \lambda_{i 2}, \ldots, \lambda_{i m}\right) \in R_{+}^{m}, i=1,2, \ldots, n$.

Then the Lagrangian dual problem is defined as the following multiobjective programming problem:

$$
\begin{array}{lll}
(\mathrm{DMP}) & \max _{\operatorname{lex}} & \phi(\Lambda) \\
& \text { s.t. } & \Lambda \in \mathscr{L}^{+}\left(R^{m}, R^{n}\right),
\end{array}
$$

where $\phi(\Lambda)=\min _{\operatorname{lex}}\{L(x, \Lambda): x \in X\}$.

Definition 6. (i) A point $\bar{x} \in E$ is said to be a lexicographic minimal solution to (MP) if $\bar{x} \in E$ and $f(\bar{x}) \leq_{\text {lex }} f(x)$, for all $x \in E$. By $\min _{\text {lex }} E$ and $\min _{\text {lex }} f(E)$ denote the set of all lexicographic minimal solutions and the lexicographic minimal value to (MP), respectively.

(ii) A point $\bar{x} \in E$ is said to be a strong minimal solution to (MP) if $\bar{x} \in E$ and $f(\bar{x}) \in f(x)-R_{+}^{n}$, for all $x \in E$; that is, $f_{i}(\bar{x}) \leq f_{i}(x)$, and $x \in E$, and $i=1,2, \ldots, n$. The set of all strong minimal solutions to (MP) is denoted by $\min _{R_{+}^{n}} f(E)$.

Remark 7. (1) From [14], it is easy to verify that (i) of Definition 6 is equivalent and refers to the following concept: a point $\bar{x} \in E$ is said to be a lexicographic minimal solution to (MP) if $\bar{x} \in E$ and $\left\{f(\bar{x})-C_{\text {lex }} \backslash\{0\}\right\} \cap f(E)=\emptyset$.

(2) If $\bar{x} \in E$ is a strong solution to (MP), then $\bar{x}$ is a lexicographic solution to (MP). However, the converse generally is not valid. 


\section{Duality Results}

The following result shows that, with respect to lexicographic order, the objective value of any feasible point to the dual problem (DMP) is less than or equal to the objective value of any feasible point to the primal problem (MP).

Theorem 8 (weak duality theorem). Consider the primal problem (MP) given by (3) and its Lagrangian dual problem (DMP) given by (7). Let $x$ be a feasible point of (MP); that is, $x \in X$ and $g(x) \in-R_{+}^{m}$. Also, let $\Lambda$ be a feasible point of (DMP); that is, $\Lambda \in \mathscr{L}^{+}\left(R^{m}, R^{n}\right)$. Then

$$
f(x) \geq_{l e x} \phi(\Lambda) .
$$

Proof. Since $x \in X, g(x) \in-R_{+}^{m}$, and $\Lambda \in \mathscr{L}^{+}\left(R^{m}, R^{n}\right)$, we obtain that $\Lambda g(x) \in-R_{+}^{n} \subset-C_{\text {lex }}$; that is, $\Lambda g(x) \leq_{\operatorname{lex}} 0_{n}$. Then, it follows from the definition of $\phi(\Lambda)$ that

$$
\begin{aligned}
\phi(\Lambda) & =\min _{\operatorname{lex}}\{f(\tilde{x})+\Lambda g(\tilde{x}): \tilde{x} \in X\} \\
& \leq_{\operatorname{lex}} f(x)+\Lambda g(x) \leq_{\operatorname{lex}} f(x),
\end{aligned}
$$

and the proof is complete.

As a corollary of the previous theorem, we have the following result.

Corollary 9. Assume that $\max _{\text {lex }}\left\{\phi(\Lambda): \Lambda \in L^{+}\left(R^{n}, R^{m}\right) \neq \emptyset\right.$. Then,

$$
\begin{aligned}
\min _{\text {lex }} & \left\{f(x): g(x) \in-R_{+}^{m}, x \in X\right\} \\
& \geq_{\text {lex }} \max _{\text {lex }}\left\{\phi(\Lambda): \Lambda \in L^{+}\left(R^{n}, R^{m}\right)\right\} .
\end{aligned}
$$

Before stating the Lagrangian multiplier rule to (MP) in lexicographic sense, we need the following result.

Lemma 10. Assume that $\bigcap_{i=1}^{n} E_{i} \neq \emptyset$. A point $\bar{x} \in E$ is a lexicographic solution for $(M P)$ if and only if $\bar{x} \in E$ is a strong solution for (MP).

Proof. If $\bar{x}$ is a lexicographic solution for (MP), then $f(\bar{x}) \in$ $\min _{\text {lex }} f(E)$. Assume that $\bigcap_{i=1}^{n} E_{i} \neq \emptyset$. Then each $\hat{x} \in \bigcap_{i=1}^{n} E_{i}$ is a strong solution for (MP), which implies that $f(\widehat{x}) \epsilon$ $\min _{\text {lex }} f(E)$. Since $\in \min _{\text {lex }} f(E)$ is singleton, we have $f(\widehat{x})=$ $f(\bar{x})$. That is, $\bar{x}$ is a strong solution for (MP).

Conversely, suppose that $\bar{x}$ is a lexicographic solution for (MP). Since $\bigcap_{i=1}^{n} E_{i} \neq \emptyset$, there exists $\hat{x} \in E$ which is a strong solution for (MP). By induction, we can show that $f(\bar{x})=f(\widehat{x})$ which means that $\bar{x}$ is a strong solution for (MP). Let $D_{k}=\left\{x \in D_{k-1}: f_{k}(x) \leq f_{k}(y), \forall y \in D_{k-1}\right\}$ for $k=1,2, \ldots, n$ and $D_{0}=E$. Obviously, $D_{n} \subset D_{n-1} \subset \cdots \subset D_{0}$. Noting that $\bar{x}$ is a lexicographic solution for (MP), we have $\bar{x} \in D_{n}$. Then, $\bar{x} \in D_{1}$; that is,

$$
f_{1}(x) \geq f_{1}(\bar{x}), \quad \forall x \in D_{0} .
$$

Taking $x=\widehat{x}$ in the above inequality, we get

$$
f_{1}(\widehat{x}) \geq f_{1}(\bar{x}) \text {. }
$$

On the other hand, $f_{1}(\bar{x}) \geq f_{1}(\widehat{x})$ since $\widehat{x} \in D_{0}$ is a strong solution and $\bar{x} \in X_{n} \subset D_{0}$. Therefore, $f_{1}(\bar{x})=f_{1}(\hat{x})$ and $\widehat{x} \in D_{1}$. Suppose that $f_{i}(\bar{x})=f_{i}(\widehat{x})$ for $i=1,2, \ldots, n-1$. Obviously, $\bar{x} \in D_{n-1}$. Similarly, we can verify that $f_{n}(\bar{x})=$ $f_{n}(\hat{x})$ and $\hat{x} \in D_{n}$. The proof is complete.

Theorem 11 (Lagrangian multiplier rule). Suppose that the following conditions are satisfied:

(i) $X$ is a nonempty convex subset of $R^{\ell}$;

(ii) $f: X \rightarrow R^{n}$ is a $R_{+}^{n}$-convex vector function; that is, $f_{i}: X \rightarrow R, i=1,2, \ldots, n$, are convex functions;

(iii) $g: X \rightarrow R^{m}$ is a $R_{+}^{m}$-convex vector function; that is, $g_{j}: X \rightarrow R, j=1,2, \ldots, m$, are convex functions;

(iv) the Slater constraint qualification is satisfied; that is, there exists $x_{0} \in X$ such that $g\left(x_{0}\right) \in-$ int $R_{+}^{m}$, where int $R_{+}^{m}$ is the topology interior of $R_{+}^{m}$;

(v) $\bigcap_{i=1}^{n} E_{i} \neq \emptyset$.

If $\bar{x}$ is a lexicographic solution to (MP), then there exists $\bar{\Lambda} \epsilon$ $\mathscr{L}^{+}\left(R^{m}, R^{n}\right)$ such that

$$
\begin{gathered}
f(\bar{x})=\min _{\text {lex }}\{f(x)+\bar{\Lambda} g(x): x \in E\}, \\
\bar{\Lambda} g(\bar{x})=0_{n} .
\end{gathered}
$$

Proof. Let $\bar{x}$ be a lexicographic solution of the problem (MP). By Lemma $10, \bar{x}$ is a strong solution for (MP). Then, for any $x \in E:=\left\{x \in X: g(x) \in-R_{+}^{m}\right\}$,

$$
\begin{gathered}
f_{1}(x) \geq f_{1}(\bar{x}), \\
f_{2}(x) \geq f_{2}(\bar{x}), \\
\vdots \\
f_{n}(x) \geq f_{n}(\bar{x}) .
\end{gathered}
$$

Then, the inequality system $f_{1}(x)<f_{1}(\bar{x}), g(x) \in-R_{+}^{m}$ for some $x \in X$ has no solution. Define the following set:

$$
\begin{aligned}
S_{1}:= & \left\{(p, q) \in R \times R^{m}: f_{1}(x)-f_{1}(\bar{x})\right. \\
& \left.<p, g(x) \in q-R_{+}^{m}, \text { for some } x \in X\right\} .
\end{aligned}
$$

The set $S_{1}$ is convex subset since $X, f_{1}$, and $g$ are convex. Since the above inequality system has no solution, we have $\left(0,0_{m}\right) \notin$ $S_{1}$. By the standard separation theorem, there exists a nonzero vector $\left(\widehat{\mu}_{1}, \widehat{\lambda}_{1}\right)$ such that

$$
\widehat{\mu}_{1}\left(f_{1}(x)-f_{1}(\bar{x})\right)+\widehat{\lambda}_{1}^{T} g(x) \geq 0, \quad \forall x \in X .
$$

That is,

$$
\widehat{\mu}_{1} f_{1}(x)+\widehat{\lambda}_{1}^{T} g(x) \geq \widehat{\mu}_{1} f_{1}(\bar{x}), \quad \forall x \in X .
$$

Now, fix an $x \in X$. From the definition of $S_{1}$, we have that $p$ and $q$ can be arbitrarily large. In order to satisfy (17), we must have $\left(\widehat{\mu}_{1}, \widehat{\lambda}_{1}\right) \in R_{+} \times R_{+}^{m}$. We will next show that $\widehat{\mu}_{1}>0$. 
On the contrary, suppose that $\widehat{\mu}_{1}=0$. By the Slater constraint qualification, there exists $x_{0} \in X$ such that $g\left(x_{0}\right) \in-$ int $R_{+}^{m}$. Then, letting $x=x_{0}$ in (17), we have $\widehat{\lambda}_{1} g\left(x_{0}\right) \geq 0$. But, since $g\left(x_{0}\right) \in-$ int $R_{+}^{m}$ and $\hat{\lambda}_{1} \in R_{+}^{m}, \hat{\lambda}_{1} g\left(x_{0}\right) \geq 0$ is only possible if $\hat{\lambda}_{1}=0_{m}$. Thus, it has been shown that $\left(\widehat{\mu}_{1}, \hat{\lambda}_{1}\right)=\left(0,0_{m}\right)$, which is a contradiction. obtain

After dividing (17) by $\widehat{\mu}_{1}$ and denoting $\bar{\lambda}_{1}=\widehat{\lambda}_{1} / \widehat{\mu}_{1}$, we

$$
f_{1}(x)+\bar{\lambda}_{1}^{T} g(x) \geq f_{1}(\bar{x}), \quad \forall x \in X
$$

From (18), letting $x=\bar{x}$, we get $\bar{\lambda}_{1}^{T} g(\bar{x}) \geq 0$. Since $g(\bar{x}) \in-R_{+}^{m}$ and $\bar{\lambda}_{1} \in R_{+}^{m}$, we obtain $\bar{\lambda}_{1}^{T} g(\bar{x})=0$.

Similarly, we can show that there exist $\bar{\lambda}_{2}, \bar{\lambda}_{3}, \ldots, \bar{\lambda}_{n} \in R_{+}^{m}$ such that, for any $x \in X$,

$$
\begin{gathered}
f_{2}(x)+\bar{\lambda}_{2}^{T} g(x) \geq f_{2}(\bar{x}), \quad \bar{\lambda}_{2}^{T} g(\bar{x})=0, \\
f_{3}(x)+\bar{\lambda}_{3}^{T} g(x) \geq f_{3}(\bar{x}), \quad \bar{\lambda}_{3}^{T} g(\bar{x})=0, \\
\vdots \\
f_{n}(x)+\bar{\lambda}_{n}^{T} g(x) \geq f_{n}(\bar{x}), \quad \bar{\lambda}_{n}^{T} g(\bar{x})=0 .
\end{gathered}
$$

Set $\bar{\Lambda}=\left(\bar{\lambda}_{1}, \bar{\lambda}_{2}, \ldots, \bar{\lambda}_{n}\right)^{T}$. Then, it follows from $R_{+}^{n} \subset C_{\text {lex }}$, (18), and (19) that

$$
\begin{gathered}
f(x)+\bar{\Lambda} g(x) \in f(\bar{x})+R_{+}^{n} \in f(\bar{x})+C_{\text {lex }}, \quad \forall x \in X, \\
\bar{\Lambda} g(\bar{x})=0_{n} .
\end{gathered}
$$

Namely,

$$
\begin{gathered}
f(x)+\bar{\Lambda} g(x) \geq_{\operatorname{lex}} f(\bar{x}), \quad \forall x \in X, \\
\bar{\Lambda} g(\bar{x})=0_{n} .
\end{gathered}
$$

Thus, (21) and weak duality theorem (Theorem 8) together yield that

$$
f(\bar{x})=\min _{\text {lex }}\{f(x)+\bar{\Lambda} g(x): x \in E\}
$$

And the proof is complete.

Now, we give an example to illustrate that Theorem 11 is applicable.

Example 12. Let $X=[-2,2] \subset R ; g: X \rightarrow R$ is defined by $g(x)=x^{2}-1$ and $f=\left(f_{1}, f_{2}\right)^{T}: X \rightarrow R^{2}$ is defined by

$$
\begin{aligned}
f_{1}(x)= \begin{cases}-2 x+1, & \text { if }-2 \leq x<-1, \\
3, & \text { if }-1 \leq x \leq 1, \\
2 x+1, & \text { if } 1<x \leq 2,\end{cases} \\
f_{2}(x)=(x-2)^{2}+1
\end{aligned}
$$

Consider the following convex multiobjective programming problem: $\min _{\mathrm{lex}}\{f(x): g(x) \leq 0, x \in X\}$. Direct computation shows that $E=\{x \in x: g(x) \leq 0\}=$ $[-1,1], E_{1}=[-1,1]$, and $E_{2}=\{1\}$. Obviously, $\bar{x}=1 \epsilon$ $E_{1} \cap E_{2}$ is a lexicographic solution of the multiobjective programming problem. Therefore, Theorem 11 is applicable. Indeed, by directly computing, there exists $\bar{\Lambda}=(0,1)^{T}$ such that

$$
\begin{gathered}
\min _{\operatorname{lex}}\{f(x)+\bar{\Lambda} g(x): x \in X\}=f(\bar{x})=(3,-1)^{T}, \\
\bar{\Lambda} g(\bar{x})=0_{2} .
\end{gathered}
$$

From Theorem 11, we get a sufficient condition for the existence of Lagrangian multiplier rule for the problem (MP). But this is not a necessary condition, as the following example shows.

Example 13. Let $X \subset R$, and $g(x)$ is given as in Example 12 . Let $f=\left(f_{1}, f_{2}\right): X \rightarrow R^{2}$ be defined by

$$
\begin{gathered}
f_{1}(x)=(x-2)^{2}+1, \\
f_{2}(x)=x .
\end{gathered}
$$

Clearly, the following problem:

$$
\min _{\operatorname{lex}}\{f(x): g(x) \leq 0, x \in X\}
$$

is a convex multiobjective programming. It follows from direct computation that $E=[-1,1] E_{1}=\{1\}$, and $E_{2}=\{-1\}$, and $\bar{x}=1$ is a unique lexicographic solution for (MP). Thus, the assumption (v) of Theorem 11 is not satisfied. However, we can verify that there exists $\bar{\Lambda}=(1,0)^{T}$ such that, for any $x \in E$,

$$
\begin{gathered}
\min _{\operatorname{lex}}\{f(x)+\bar{\Lambda} g(x): x \in X\}=f(\bar{x})=(2,1)^{T}, \\
\bar{\Lambda} g(\bar{x})=0_{2} .
\end{gathered}
$$

In order to obtain another sufficient condition for the existence of Lagrangian multiplier rule, we consider the following assumptions.

Theorem 14 (Lagrangian multiplier rule). Assume that all conditions of Theorem 11 are satisfied except for the hypothesis (v) of Theorem 11 which is replaced by the following hypothesis:

(v') $f_{1}: X \rightarrow R$ is a strict convex function; that is, for any $x_{1}, x_{2} \in X$ with $x_{1} \neq x_{2}$ and $\theta \in(0,1), f_{1}\left(\theta x_{1}+\right.$ $\left.(1-\theta) x_{2}\right)<\theta f_{1}\left(x_{1}\right)+(1-\theta) f_{1}\left(x_{2}\right)$.

If $\bar{x}$ is a lexicographic solution to $(M P)$, then there exists $\bar{\Lambda} \in L^{+}\left(R^{m}, R^{n}\right)$ such that

$$
\begin{gathered}
f(\bar{x})=\min _{\text {lex }}\{f(x)+\bar{\Lambda} g(x): x \in E\}, \\
\bar{\Lambda} g(\bar{x})=0_{n} .
\end{gathered}
$$


Proof. Since $X$ is convex and $g(x)$ is $R_{+}^{m}$-convex, the set $E=$ $\left\{x \in X: g(x) \in-R_{+}^{m}\right\}$ is convex. Noting that $f_{1}(x)$ is strict convex, we obtain $E_{1}=\left\{\bar{x} \in E: f_{1}(\bar{x})=\min f_{1}(E)\right\}$ is singleton. Obviously, $\bar{x} \in E_{1}=\{\bar{x}\}$ is a lexicographic solution to (MP). Following the same arguments as in Theorem 11, there exists $\bar{\lambda} \in R_{+}^{m}$ such that $f_{1}(\bar{x})=\min \left\{f_{1}(x)+\bar{\lambda}^{T} g(x)\right.$ : $x \in X\}, \bar{\lambda}^{T} g(\bar{x})=0$. Since $g(x)$ is $R_{+}^{m}$-convex and $\bar{\lambda} \epsilon$ $R_{+}^{m}$, we obtain that $\bar{\lambda}^{T} g(x)$ is a convex function $\left(R^{+}\right.$-convex function). By hypothesis $\left(\mathrm{v}^{\prime}\right), f_{1}(x)+\bar{\lambda}^{T} g(x)$ is a strict convex function. Thus, the solution to $\min \left\{f_{1}(x)+\bar{\lambda}^{T} g(x): x \in X\right\}$ is also singleton. Let $\bar{\lambda}_{1}=\bar{\lambda}, \bar{\lambda}_{2}=\cdots=\bar{\lambda}_{n}=0_{m}$ in Theorem 11. Therefore, $\bar{x}$ is also a lexicographic solution to $\min _{\text {lex }}\left\{f(x)+\bar{\Lambda}^{T} g(x): x \in X\right\}$ and $\bar{\Lambda} g(\bar{x})=0_{n}$. It completes the proof.

Remark 15. The hypothesis $\left(\mathrm{v}^{\prime}\right)$ in Theorem 14 is redundant if $f$ is a real-valued function. Under this case, the hypothesis (v) in Theorem 11 always holds.

From Theorems 8 and 11, we have the following strong duality theorem. The following result shows that, under suitable assumptions, there is no duality gap between the primal and dual lexicographic optimal objective function values.

Theorem 16 (strong duality theorem). Assume that $\max _{\text {lex }}\left\{\phi(\Lambda): \Lambda \in L^{+}\left(R^{n}, R^{m}\right) \neq \emptyset\right.$. If all conditions of Theorem 11 or Theorem 14 are satisfied, then

$$
\begin{aligned}
\min _{\text {lex }}\left\{f(x): g(x) \in-R_{+}^{m}, x \in X\right\} \\
=\max _{\text {lex }}\left\{\phi(\Lambda): \Lambda \in L^{+}\left(R^{n}, R^{m}\right)\right\} .
\end{aligned}
$$

And there exists $\bar{\Lambda}$ such that $\bar{\Lambda} g(\bar{x})=0_{n}$, where $\bar{x} \in\{x \in X$ : $\left.f(x)=\min _{\text {lex }} f(E)\right\}$.

The following example is to illustrate that there is no duality gap if all conditions of Theorem 16 are satisfied.

Example 17. Let $X \subset R, g(x)$ is given as in Example 12. Let $f(x)$ be defined by

$$
\begin{gathered}
f_{1}(x)=(x-2)^{2}+1, \\
f_{2}(x)=-x .
\end{gathered}
$$

Direct computation shows that

$$
\begin{aligned}
\min _{\operatorname{lex}}\left\{f(x): g(x) \in-R_{+}^{m}, x \in X\right\} \\
=\max _{\operatorname{lex}}\left\{\phi(\Lambda): \Lambda \in L^{+}\left(R^{n}, R^{m}\right)\right\} \\
=(2,-1)^{T} \\
=f(1) .
\end{aligned}
$$

And there exists $\bar{\Lambda}=(1,1 / 2)^{T}$ or $(1,0)^{T}$ such that $\bar{\Lambda} g(1)=$ $0_{2}$.

\section{Lexicographic Saddle Point}

Now, we introduce the notion of lexicographic saddle point for the vector-valued Lagrangian map $L(\cdot, \cdot)$ in terms of lexicographic order and give some optimality conditions.

Definition 18. A pair $(\bar{x}, \bar{\Lambda}) \in E \times \mathscr{L}\left(R^{n}, R^{m}\right)$ is said to be a lexicographic saddle point for the vector-valued Lagrangian function $L(x, \Lambda)$ if, for all $x \in E$ and $\Lambda \in \mathscr{L}^{+}\left(R^{m}, R^{n}\right)$,

$$
L(\bar{x}, \Lambda) \leq_{\operatorname{lex}} L(\bar{x}, \bar{\Lambda}) \leq_{\operatorname{lex}} L(x, \bar{\Lambda}) .
$$

By Theorem 11 or Theorem 14, we directly have the following result.

Theorem 19. Suppose that all conditions of Theorem 11 or Theorem 14 are satisfied. If $\bar{x}$ is a lexicographic solution to (MP), then there exists $\bar{\Lambda} \in \mathscr{L}^{+}\left(R^{m}, R^{n}\right)$ such that $(\bar{x}, \bar{\Lambda})$ is a lexicographic saddle point of the vector Lagrangian function $L(x, \Lambda)$.

Thus, we have verified the existence of a lexicographic saddle point for the vector-valued Lagrangian function $L(x, \Lambda)$ under the appropriate conditions. We conclude this result by showing that the saddle point condition is sufficient for optimality for problem (MP).

Theorem 20. If $(\bar{x}, \bar{\Lambda}) \in E \times \mathscr{L}\left(R^{m}, R^{n}\right)$ is a lexicographic saddle point for the vector-valued Lagrangian function $L(x, \Lambda)$, then $\bar{x}$ is a lexicographic solution for $(M P)$, and $\bar{\Lambda} g(\bar{x})=0_{n}$.

Proof. Assume that $(\bar{x}, \bar{\Lambda})$ is a lexicographic saddle point of $L(x, \Lambda)$. Then, from the first inequality of (33), we get

$$
f(\bar{x})+\bar{\Lambda} g(\bar{x}) \geq_{\operatorname{lex}} f(\bar{x})+\Lambda g(\bar{x}), \quad \forall \Lambda \in \mathscr{L}^{+}\left(R^{m}, R^{n}\right) .
$$

This implies that

$$
\bar{\Lambda} g(\bar{x}) \geq_{\operatorname{lex}} \Lambda g(\bar{x}), \quad \forall \Lambda \in \mathscr{L}^{+}\left(R^{m}, R^{n}\right) .
$$

We claim that $g(\bar{x}) \in-R_{+}^{m}$. Otherwise, we suppose that $g(\bar{x}) \notin-R_{+}^{m}$. Since $\Lambda \in \mathscr{L}^{+}\left(R^{m}, R^{n}\right)$ can be taken arbitrarily to be large, $\Lambda g(\bar{x})$ can be large enough, which contradicts (35).

Therefore, we also get

$$
\bar{\Lambda} g(\bar{x}) \in-R_{+}^{n} \subset-C_{\text {lex }}
$$

since $g(\bar{x}) \in-R_{+}^{m}$ and $\bar{\Lambda} \in \mathscr{L}^{+}\left(R^{m}, R^{n}\right)$. This implies that

$$
\bar{\Lambda} g(\bar{x}) \leq_{\operatorname{lex}} 0_{n} .
$$

On the other hand, by taking $\Lambda=0$ in (35), we can get

$$
\bar{\Lambda} g(\bar{x}) \geq_{\operatorname{lex}} 0_{n} .
$$

Noting the reflexivity of the lexicographic order $\leq_{\text {lex }}$, (37) and (38) together yields

$$
\bar{\Lambda} g(\bar{x})=0_{n}
$$


Since $g(\bar{x}) \in-R_{+}^{m}, \bar{x}$ is a feasible point of (MP). Let $x$ be any feasible point of (MP); that is, $g(x) \in-R_{+}^{m}$. Then, it follows from the second inequality of (33) and (39) that

$$
\begin{aligned}
f(\bar{x}) & =f(\bar{x})+\bar{\Lambda} g(\bar{x}) \\
& \leq_{\operatorname{lex}} f(x)+\bar{\Lambda} g(x) \leq_{\operatorname{lex}} f(x),
\end{aligned}
$$

which implies that $\bar{x}$ is a lexicographic solution to (MP).

\section{Acknowledgments}

The authors would like to express their deep gratitude to the anonymous referee and the associate editor for their valuable comments and suggestions which helped improve the paper. This research was partially supported by the National Natural Science Foundation of China (Grant no. 11201509).

\section{References}

[1] H. W. Corley, "Duality theory for maximizations with respect to cones," Journal of Mathematical Analysis and Applications, vol. 84, no. 2, pp. 560-568, 1981.

[2] H. W. Corley, "Existence and lagrangian duality for maximizations of set-valued functions," Journal of Optimization Theory and Applications, vol. 54, no. 3, pp. 489-501, 1987.

[3] P. C. Fishburn, "Lexicographic orders, utilities and decision rules: a survey," Management Science, vol. 20, pp. 1442-1471, 1974.

[4] W.-S. Hsia and T. Y. Lee, "Lagrangian function and duality theory in multiobjective programming with set functions," Journal of Optimization Theory and Applications, vol. 57, no. 2, pp. 239-251, 1988.

[5] E. Hernández and L. Rodríguez-Marín, "Lagrangian duality in set-valued optimization," Journal of Optimization Theory and Applications, vol. 134, no. 1, pp. 119-134, 2007.

[6] J. Jahn, Mathematical Vector Optimization in Partially Ordered Linear Spaces, vol. 31 of Methoden und Verfahren der Mathematischen Physik, Peter Lang, Frankfurt, Germany, 1986.

[7] Z.-F. Li and G.-Y. Chen, "Lagrangian multipliers, saddle points, and duality in vector optimization of set-valued maps," Journal of Mathematical Analysis and Applications, vol. 215, no. 2, pp. 297-316, 1997.

[8] Z. F. Li and S. Y. Wang, "Lagrange multipliers and saddle points in multiobjective programming," Journal of Optimization Theory and Applications, vol. 83, no. 1, pp. 63-81, 1994.

[9] W. Song, "Lagrangian duality for minimization of nonconvex multifunctions," Journal of Optimization Theory and Applications, vol. 93, no. 1, pp. 167-182, 1997.

[10] Y. Sawaragi, H. Nakayama, and T. Tanino, Theory of Multiobjective Optimization, vol. 176 of Mathematics in Science and Engineering, Academic Press, Orlando, Fla, USA, 1985.

[11] T. Tanino and Y. Sawaragi, "Duality theory in multiobjective programming," Journal of Optimization Theory and Applications, vol. 27, no. 4, pp. 509-529, 1979.

[12] I. V. Konnov, "On lexicographic vector equilibrium problems," Journal of Optimization Theory and Applications, vol. 118, no. 3 , pp. 681-688, 2003.

[13] X. B. Li, S. J. Li, and Z. M. Fang, "A minimax theorem for vectorvalued functions in lexicographic order," Nonlinear Analysis:
Theory, Methods \& Applications, vol. 73, no. 4, pp. 1101-1108, 2010.

[14] M. M. Mäkelä and Y. Nikulin, "On cone characterizations of strong and lexicographic optimality in convex multiobjective optimization," Journal of Optimization Theory and Applications, vol. 143, no. 3, pp. 519-538, 2009.

[15] J. E. Martínez-Legaz, "Lexicographical order, inequality systems and optimization," in System Modelling and Optimization, P. Thoft-Christensen, Ed., vol. 59 of Lecture Notes in Control and Information Sciences, pp. 203-212, Springer, Berlin, Germany, 1984.

[16] J.-E. Martínez-Legaz, "Lexicographical order and duality in multiobjective programming," European Journal of Operational Research, vol. 33, no. 3, pp. 342-348, 1988.

[17] V. V. Podinovskii and V. M. Gavrilov, Optimization with Respect to Sequentially Applied Criteria, Sovetskoe Radio, Moscow, Russia, 1976, (Russian).

[18] L. Pourkarimi and M. Zarepisheh, "A dual-based algorithm for solving lexicographic multiple objective programs," European Journal of Operational Research, vol. 176, no. 3, pp. 1348-1356, 2007.

[19] N. Popovici, "Structure of efficient sets in lexicographic quasiconvex multicriteria optimization," Operations Research Letters, vol. 34, no. 2, pp. 142-148, 2006. 


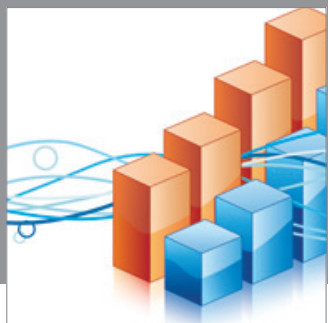

Advances in

Operations Research

mansans

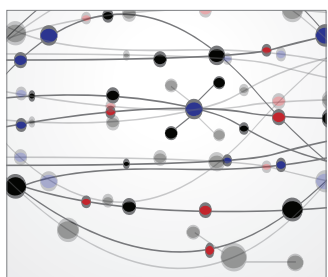

The Scientific World Journal
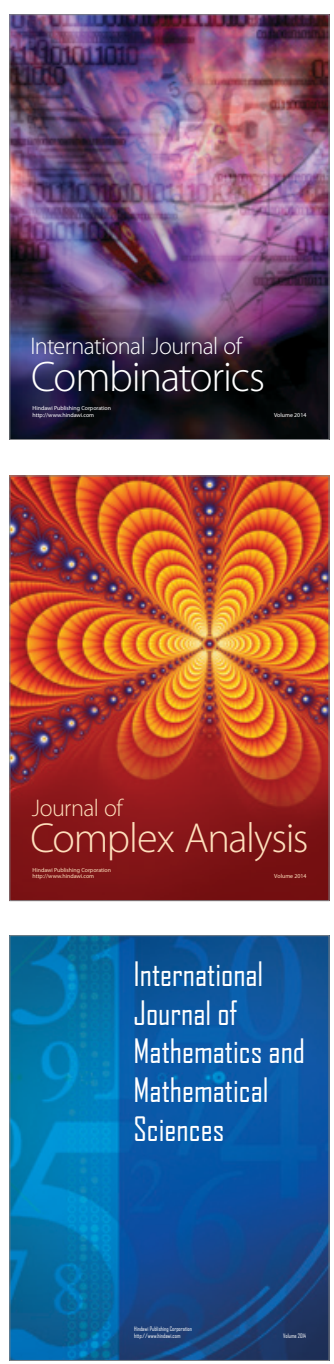
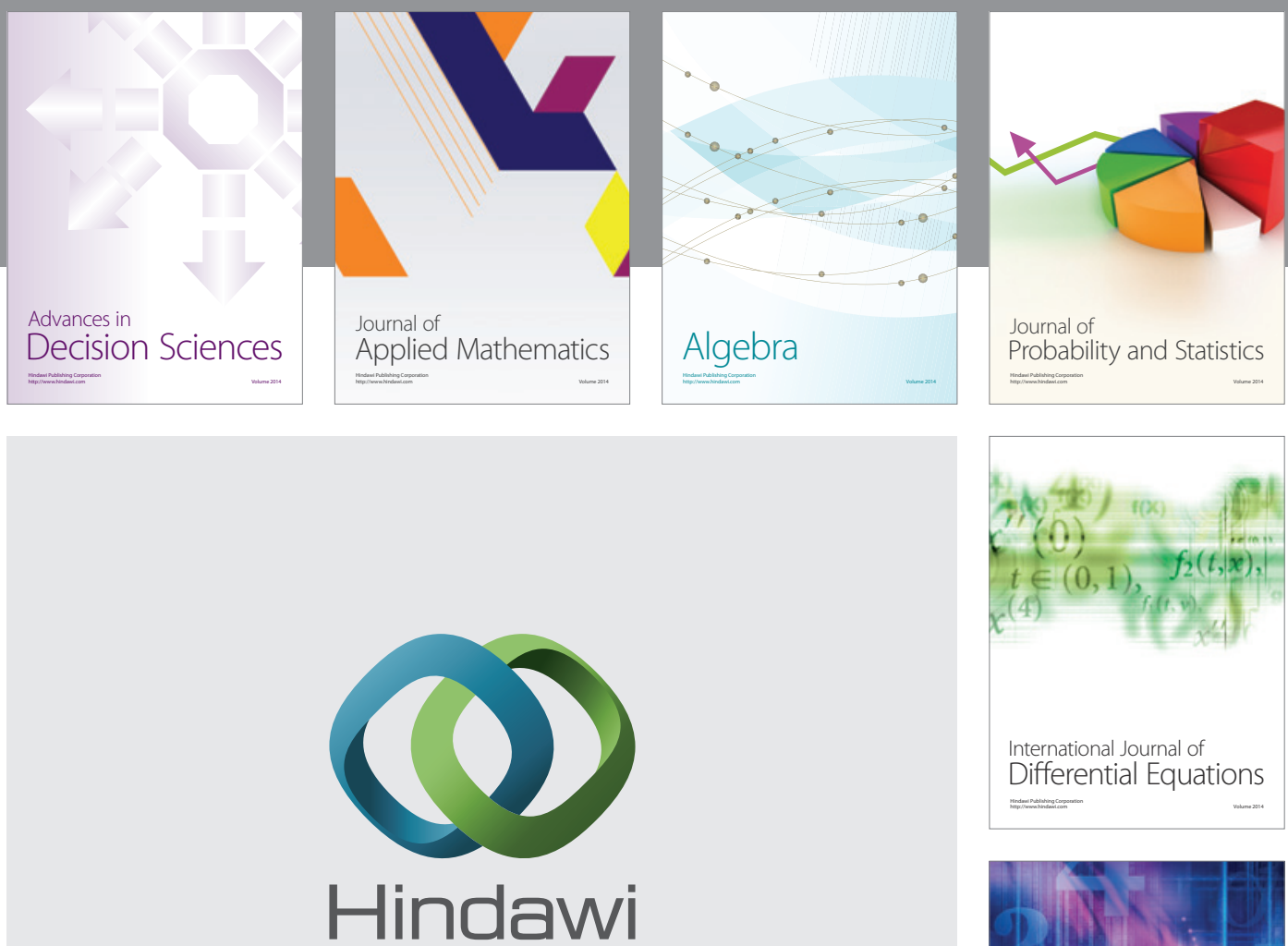

Submit your manuscripts at http://www.hindawi.com
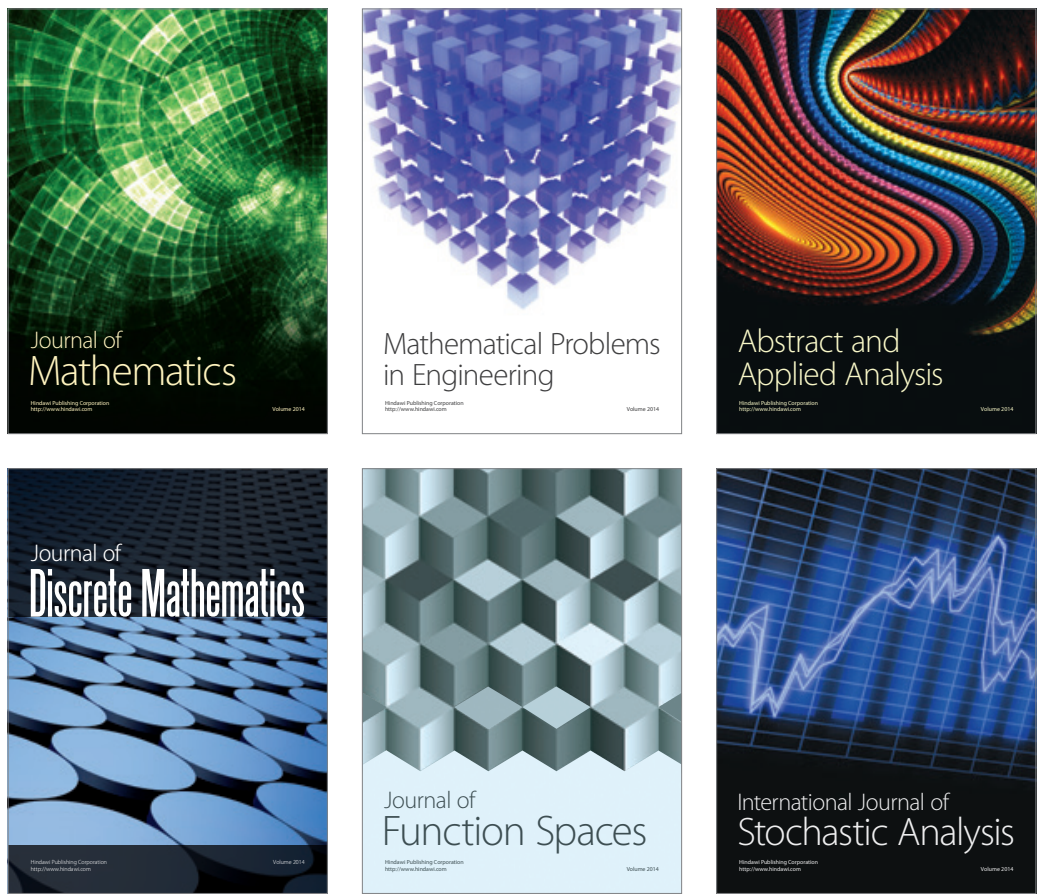

Journal of

Function Spaces

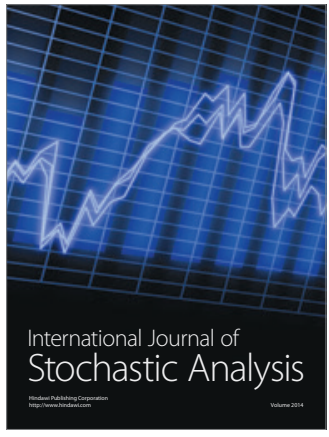

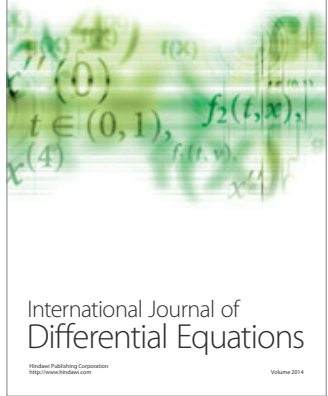
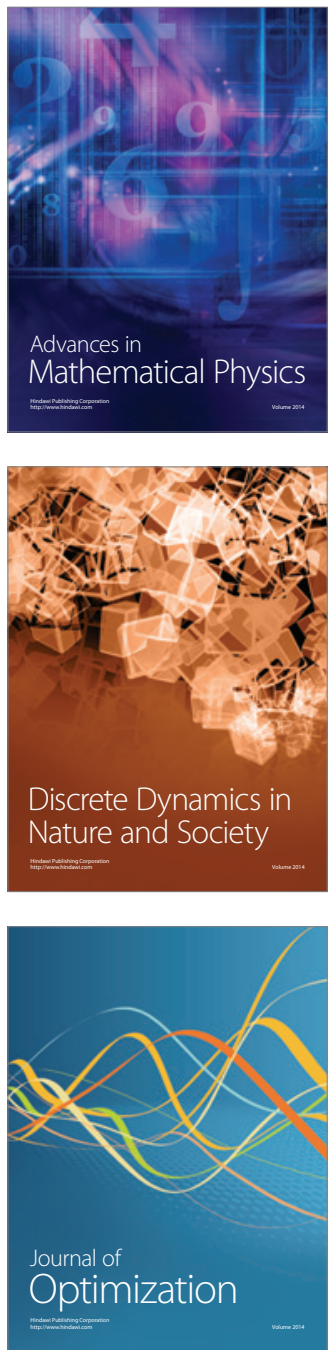